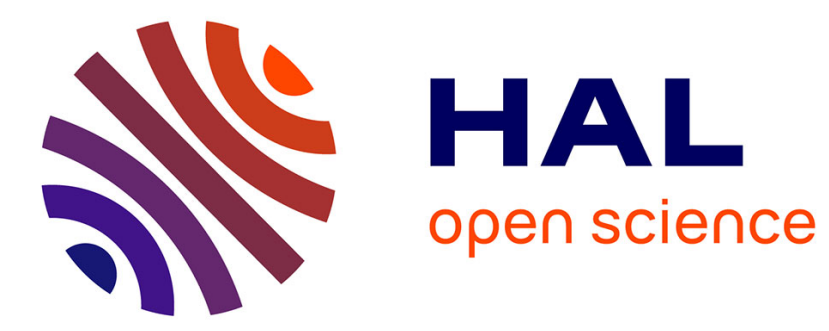

\title{
Slip lines versus shear bands: two competing localization modes
}

\author{
Félix Darve, Francois Nicot, Antoine Wautier, Jiaying Liu
}

\section{To cite this version:}

Félix Darve, Francois Nicot, Antoine Wautier, Jiaying Liu. Slip lines versus shear bands: two competing localization modes. Mechanics Research Communications, 2021, pp.1-17. 10.1016/j.mechrescom.2020.103603 . hal-03462444

\section{HAL Id: hal-03462444 \\ https://hal.inrae.fr/hal-03462444}

Submitted on 1 Dec 2021

HAL is a multi-disciplinary open access archive for the deposit and dissemination of scientific research documents, whether they are published or not. The documents may come from teaching and research institutions in France or abroad, or from public or private research centers.
L'archive ouverte pluridisciplinaire HAL, est destinée au dépôt et à la diffusion de documents scientifiques de niveau recherche, publiés ou non, émanant des établissements d'enseignement et de recherche français ou étrangers, des laboratoires publics ou privés. 
Slip lines versus shear bands: two competing localization modes

Félix DARVE $^{1}$, Francois NICOT ${ }^{2}$, Antoine WAUTIER ${ }^{3}$, Jiaying LIU ${ }^{4}$

${ }^{1}$ University Grenoble Alpes, Grenoble INP, CNRS, lab 3SR, France

${ }^{2}$ University Grenoble Alpes, INRAE, UR ETNA, Grenoble, France

3 INRAE, UR RECOVER, Aix-Marseille University, Aix en Provence, France

${ }^{4}$ State Key Laboratory of Water Resources and Hydropower Engineering Science, Wuhan University, Wuhan, China

Corresponding author Felix.Darve@grenoble-inp.fr

Tel.:+33 670799846

\begin{abstract}
Granular materials can develop two different failure modes following their initial density and the loading path: a localized mode, generally characterized by shear bands, and a diffuse mode without any macroscopic localized bands. In fact detailed analyses of experiments by tomography, of finite element computations and more recently of discrete element models (DEM) simulations show that two different localized objects co-exist at two different scales: a network of meso-slip lines appearing from the very beginning of deviatoric loading and a few macro-shear bands when approaching limit stress states. This paper aims to compare and analyze these two localized patterns, based on DEM results obtained for a given class of loading paths applied to several 2D numerical granular samples with different initial densities.

It is first shown that void ratios and granular meso-structures are the same inside shear bands and for the whole samples in case of diffuse failure. Then slip lines and shear bands are shown to be distinct localized objects. Eventually, the questions of why and how the network of meso-slip lines is sometimes bifurcating into a set of few macro-shear bands are discussed.
\end{abstract}

Keywords: granular material, slip line, shear band, strain localization, DEM, meso structures

\title{
1. Introduction
}

A general characteristic feature of plastic or damage strains (i.e. irreversible rate-independent strains) seems to be the fact that these strains localize for a given regime of deformation. Conversely, strain localization phenomenon is largely non-existent in cases of reversible strains (elastic behaviour) and of rate-dependent strains (viscous behaviour). Localized modes are commonly observed for example in metal plasticity (the so-called Lüders lines) and in geomaterials (soils, rocks and concretes) [1, 2, 
3, 23]. According to these observations, two families of localized strains co-exist. At the first regime of small deformation, there is generally a network of meso-localized strain bands (e.g. defined as microbands in [4]), while for larger strain levels, a few macro-shear bands [1] (or compaction/dilation bands) are often visible. The distinction of these structures involves two different scales of interest and for the sake of clarity it is emphasized that micro-scale refers hereafter to the grain scale, mesoscale to the assemblies of about 3 to 30 grains with associated voids and macro-scale to the continuum sample or REV scale.

For fragile materials, meso-fissures or meso-fractures resulting from damage localization form the network of meso-bands. For ductile media, this network is based on meso-shear strains resulting from plastic strain localization. Sometimes this meso-network is persistent and the failure can be characterized as "diffuse" [5,6]. This is the case for the well-known example of loose sand liquefaction, for which no shear band form. For other materials or different initial densities and loading paths, few shear bands appear close to the failure limit states.

This paper focuses on granular materials, for which it is well recognized that both diffuse and localized failure modes co-exist [9,14]. Typically, loose sands are more prone to diffuse failures than dense sands, but the loading path is also playing a major role as even a dense sand can be liquefied without shear band by a proportional strain path when it is dilatant enough [7].

Another aspect, in favor of the analysis of granular materials, is the fact that grain scale simulations can be used with confidence to simulate the overall behavior of these materials. Indeed, since several decades, the discrete element method (DEM) has become very popular for describing macroscopic granular material behavior because very realistic results are commonly obtained with few input parameters [8].

Direct numerical simulations of granular media by DEM are based on the definition of contact forces (unlike interaction potentials as done in molecular dynamics). For non-cohesive granular materials, these forces are well described by a normal elastic stiffness modulus $k_{n}$, a tangential elastic modulus $k_{t}$, and the limitation of the tangential force by a Coulombian limit characterized by a friction angle $\varphi \mathrm{g}:$

$F_{n}=k_{n} u_{n}$, where $k_{n}$ is the normal elastic stiffness and $u_{n}$ the normal granular interpenetration,

$\delta F_{t}=k_{t} \delta u_{t}$, where $k_{t}$ is the tangential elastic stiffness and $\delta u_{t}$ the incremental tangential displacement,

$$
\text { with } F_{t}+\delta F_{t} \leq F_{n} \tan \varphi_{\mathrm{g}}
$$

Steady state failure regime of granular materials is characterized by the so-called "critical states", defined by continuously growing deviatoric strain under constant stress state and constant volume [9]. So, a first question can be raised: is the critical state identical in diffuse failure for the whole sample and in localized failure for the inside of shear bands? This question will be discussed in a first part of this paper in 2D by comparing not only the void ratio for diffuse failure and in the shear band area but also, in both cases, the meso-structures, constituted by grain loops in $2 \mathrm{D}$ - that is to say grains in contact forming enclosed loops of different sizes corresponding to different numbers of grains [9]. 
Having shown that the critical state meso-structure is unique for diffuse or localized failures, a second question is arising: why for some given initial densities and loading paths the meso-network of localized bands is giving rise to few macro-shear bands? This second question is discussed in the second part of this paper.

In both sections, an important effort is paid to interpret the failure mechanisms observed by DEM within the general framework of non-associated elasto-plasticity theory.

\section{General common features of failure in granular media}

Failure modes of four different 2D granular samples with different initial void ratios $\mathrm{e}_{0}$ have been compared under drained biaxial loadings with DEM code "YADE" (see [9]). These samples are defined as follows:

- $\quad$ sample (S1): $\mathrm{e}_{0}=0.185$; see Fig.1 (a),

- $\quad$ sample (S2): $\mathrm{e}_{0}=0.200$; see Fig. 1 (b),

- $\quad$ sample (S3): $\mathrm{e}_{0}=0.246$; see Fig. 1 (c)

- $\quad$ sample (S4): $\mathrm{e}_{0}=0.287$; see Fig. 1(d).

The parameters of the simulations are the following ones (see [9]):

particle number: 25000 ; model sizes: 0.90 m - 1.35 m

particle radii range: $0.75 \mathrm{D}_{50}-1.25 \mathrm{D}_{50}$; particle density : $2600 \mathrm{~kg} / \mathrm{m}^{3}$

$\mathrm{k}_{\mathrm{n}} / \mathrm{p}_{\mathrm{a}}=10^{4} ; \mathrm{k}_{\mathrm{t}} / \mathrm{k}_{\mathrm{n}}=0.5$

intergranular friction angle $\varphi_{\mathrm{g}}=30^{\circ}$; particle wall friction angle: $0^{\circ}$

confining pressure $\mathrm{p}_{\mathrm{a}}=100 \mathrm{kPa}$; loading rate: $0.01 \mathrm{~s}^{-1}$

For these four numerical samples, the loading path is the same: only the initial densities are different. A drained biaxial compression path, with a constant lateral pressure of $100 \mathrm{kPa}^{1}$, has been chosen in order to obtain both localized and diffuse failures only by changing the initial densities but keeping the granular material, the loading path and the boundary conditions unchanged. In the present study, we define piecewise constant stress and incremental strain field maps. The incremental grain displacements are interpolated to define meso-strain tensors attached to each grain loop (we recall here that the collection of loops forms a pavement in 2D). The mean grain stresses (computed from contact forces) are averaged over the loop area to define meso-stress tensors attached to each loop. This enables the visualization of mesoscale incremental deviatoric strains presented in Fig. 1, 5 and 6. In Fig. 1 incremental deviatoric strain maps are computed at failure states [9].

Typically (S1), (S2), (S3) behave like dense granular materials with clear shear bands, while (S4) exhibits a diffuse mode of failure without any macro-shear band. For case (S1) - Fig. 1(a) - probably there are two parallel shear bands. So, by taking into account the latter remark and as it is expected

\footnotetext{
${ }^{1}$ In 2D simulations, the out of plane dimension corresponding to the maximum grain diameter is used to define stresses in $\mathrm{kPa}$.
} 
from experimental lab results, the thickness of shear bands increases when the initial density decreases (or equivalently the initial void ratio increases) [10] until the disappearance of any shear band, replaced by a diffuse mode of failure. More precisely, in all subfigures of Fig. 1, the total width of the shear band is equal to $2 \delta$. The precise definition of this length scale can be found in [9] and is based on the following steps:

- Interpolate the grain displacements to construct a continuous displacement field

- Assume a diagonal shear band

- Compute the mean strain values at a given distance $\alpha$ from the diagonal

- Fit the curve $\left\langle\varepsilon_{11}>=f(\alpha)\right.$ with a bell shape function ( $\varepsilon_{11}$ is the horizontal strain)

- The half width of the "bell” gives the $\delta$ value.

This definition of $\delta$ is thus rather empirical, but the purpose is only to have an objective criterion to distinguish between a zone inside and outside of the shear band. For the used particle size distribution (uniform radius distribution in $\left[0.75 R_{50} ; 1.25 R_{50}\right]$ ) the shear band width $2 \delta$ is between 25 and 50 times the mean grain diameter $\left(D_{50}\right)$, which seems to be reasonable according to classical views.

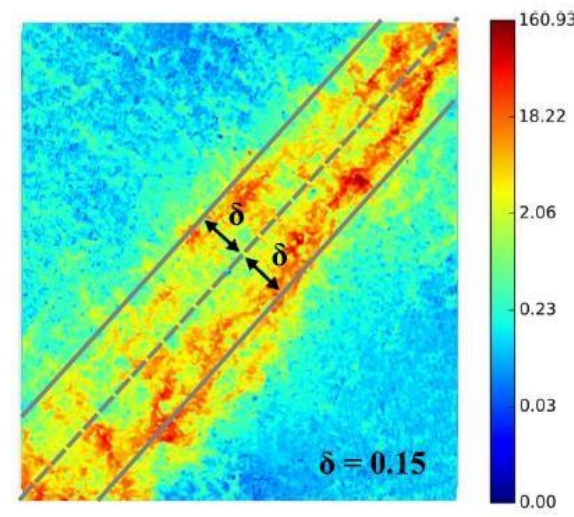

(a)

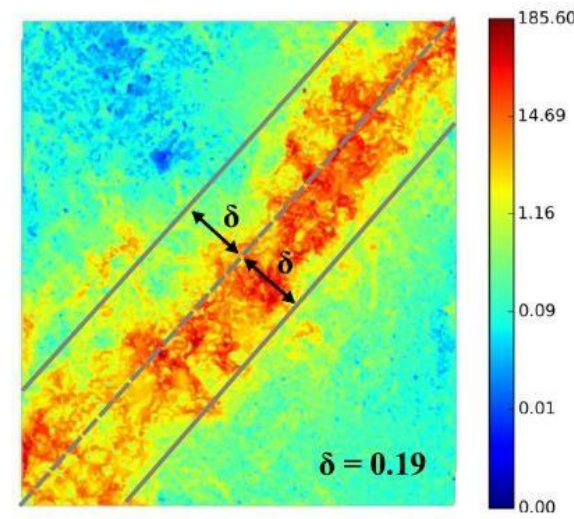

(c)

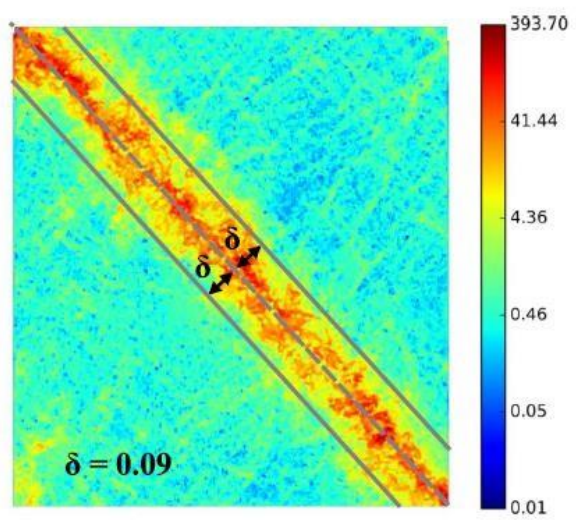

(b)

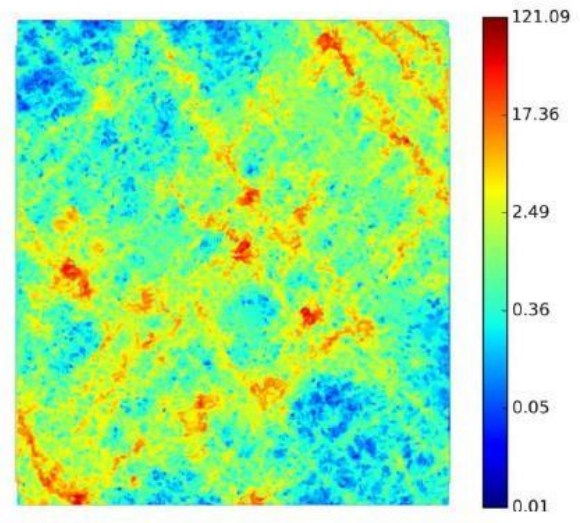

(d)

Fig. 1: 2D modelling by DEM of four numerical granular samples of four different initial densities. Maps of incremental deviatoric strains are shown at failure states. S1(a), S2(b), S3(c) correspond to dense samples with localized failure by shear bands, while S4(d) is a loose sample with a diffuse failure [9]. 
Besides, let us note that the orientation of shear band is not a material/constitutive parameter in these numerical experiments. Indeed the geometric boundary conditions are imposing a diagonal band going from corner to corner. For larger sample aspect ratios, reflecting shear bands can be observed exhibiting a competition between a direction imposed by the boundary conditions and a constitutive shear band direction. In lab experiments, the use of flexible lateral boundaries makes the shear band direction less sensitive to the boundary conditions. It has been repeatedly observed that the band orientation with respect to the major stress principal direction (the vertical direction in Fig. 1) increases with the decrease in initial density (or increase of void ratio) until $45^{\circ}$, where no shear band persists. According to experiments and classical empirical views [2], this orientation angle is approximately equal to:

$\pi / 4-(\varphi+\psi) / 4$,

where $\varphi$ is the friction angle and $\psi$ the dilatancy angle, defined in 2D by ( see Fig.2) :

$\sin \varphi=\left(\sigma_{1}-\sigma_{3}\right) /\left(\sigma_{1}+\sigma_{3}\right)$

and $\sin \psi=\left(\mathrm{d} \varepsilon_{1}+\mathrm{d} \varepsilon_{3}\right) /\left(\mathrm{d} \varepsilon_{1}-\mathrm{d} \varepsilon_{3}\right)$,

where 1 is the major stress direction and 3 the minor stress direction. The sign convention of soil mechanics is applied (positive stress in compression and positive strain in contraction).

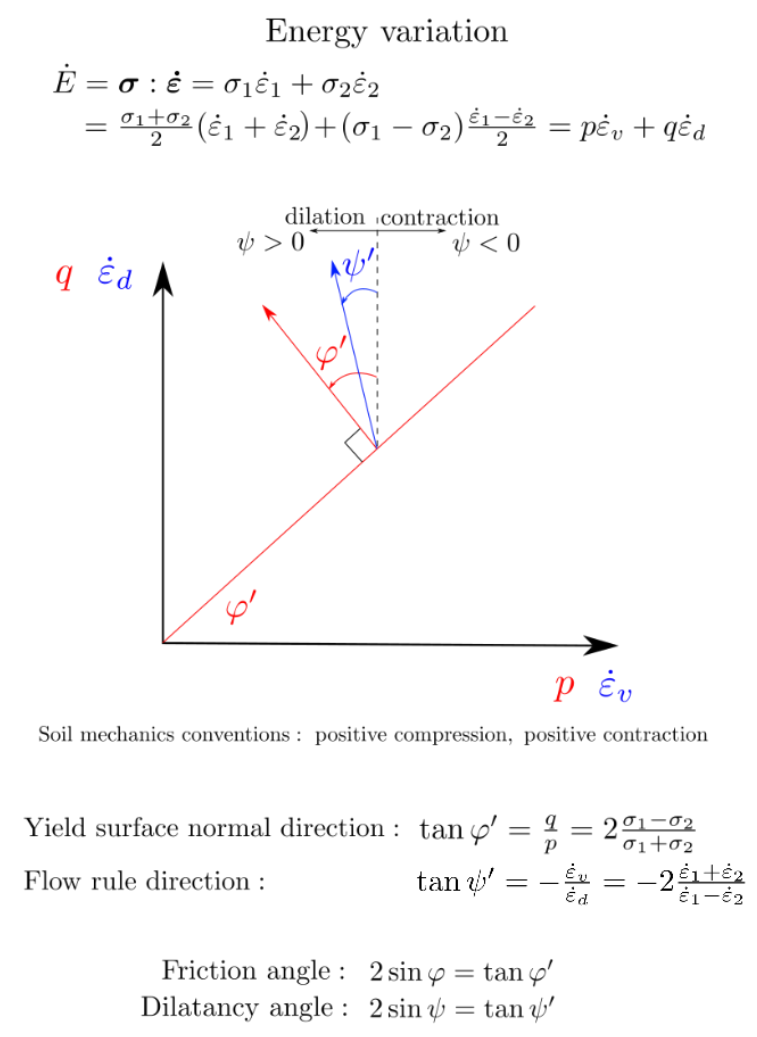

Fig. 2: Basic definitions and notations for dense and loose sands with conjugate variables with respect to energy. The dilatancy angle changes its sign from dense sands (positive) to loose ones (negative).

We can conclude that these numerical results in fig. 1 are well representative of the classical qualitative behavior of granular materials at failure as observed in lab experiments. 
In Fig.3 [9], a comparison is presented between the void ratios inside the shear bands for the three dense samples (S1, S2, S3) and in the whole sample for the loose one. The asymptotic values of these four void ratios are the same inside the shear bands, independently of the initial densities. This value corresponds to the void ratio of the whole sample in the case of a loose material with a diffuse failure. Of course, if the average values of the void ratio are computed for the whole samples in the dense cases S1, S2 and S3, the asymptotic values are fully different as shown in Fig.3. Indeed it is known from theoretical analyses that there is an elastic unloading outside shear bands just after the bifurcation point with void ratios different from the critical void ratios obtained at steady state failure inside the shear bands.

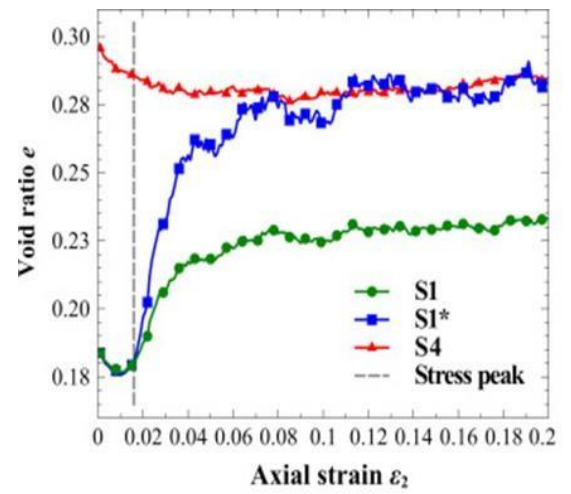

(a)

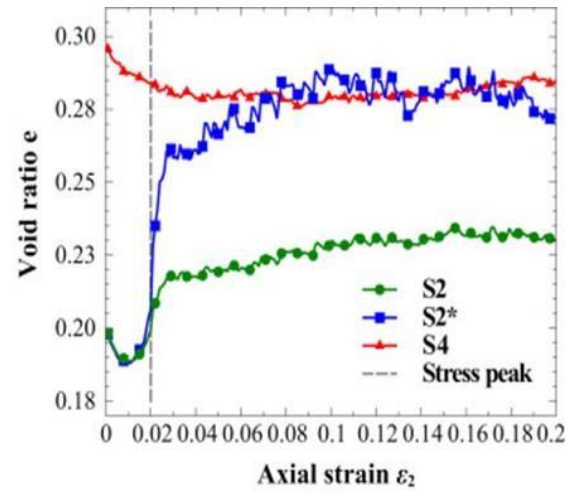

(b)

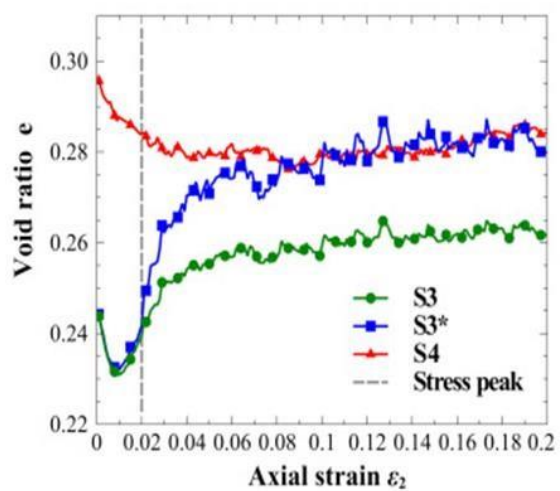

(c)

Fig. 3: comparison between the void ratios inside the shear bands (noted by ${ }^{*}$ ) for dense samples S1(a), S2(b), S3(c) with the void ratio for the whole sample of the loose specimen S4 [9].

Then in Fig. 4 [9], a comparison is shown between meso-structures inside the shear bands in case of localized failures and for the whole sample in case of diffuse failure. For that, the evolution of the relative ratios of loops composed of 3, 4, 5 and more than 6 grains are compared. These evolutions are rather intricate, since the ratio of loops with 3 grains decreases while the ratio of loops with more than 6 grains increases for dense samples. However the asymptotic values of these loop ratios are the same inside the shear bands and for the whole sample in case of diffuse failure.

Thus, a strong conclusion is emerging from these results. Not only the void ratios but also the mesostructures (characterised by the relative number of the different loops) are the same at failure 
states inside the shear bands and for the whole sample in the case of diffuse failure. In other words, granular failure structure is unique and failure inside shear bands is indeed diffuse. Nature has one unique way to develop large strains in granular materials and to reach the so-called « critical states ». This result will enable to draw a link between meso and macro localized structures in the next section.

Besides, this result is consistent with elasto-plasticity theory. Indeed, in the one hand the localization criterion (existence of shear bands) is given by the vanishing of the determinant of the acoustic tensor $[11,12,13]$ :

$$
\operatorname{det} \boldsymbol{n} . \boldsymbol{L} \cdot \boldsymbol{n}=0,
$$

where $\boldsymbol{L}$ is the four order elasto-plastic tensor linking stress and strain rates $(\dot{\boldsymbol{\sigma}}=\boldsymbol{L}: \dot{\boldsymbol{\varepsilon}})$ and $\boldsymbol{n}$ the vector normal to the shear band.

On the other hand, the necessary condition of diffuse failure corresponds to the singularity of the symmetric part of the $6 * 6$ elasto-plastic matrix $\boldsymbol{M}\left(\boldsymbol{M}=\left[M_{\alpha \beta}\right]\right.$ with $\left.\alpha, \beta=1,2, \ldots, 6\right)$ linking stress and strain vectors written with Mandel's notations [5,6,14]:

$$
\operatorname{det} \boldsymbol{M}_{\boldsymbol{s}} \leq 0
$$

According to linear algebra, condition (5) is fulfilled only when condition (6) is itself verified, while the reciprocal implication is not true for non-associated elasto-plastic materials [14].

Thus, inside shear bands, Equations (5) and (6) are automatically fulfilled, which means that diffuse failure can be observed either alone for the whole specimen or localized inside shear bands. This result has also been verified numerically by FEM [15].

However, why for some initial densities and loading paths, failure is macro-localized in rateindependent materials? Some elements of responses are given in the next section, based on the fact that shear band formation is a general phenomenon observed for plastic materials exhibiting very different micro-structures (like metals and geomaterials for example). 


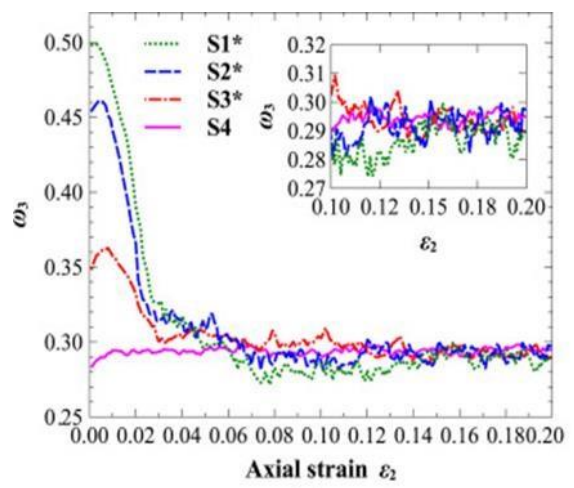

(a)

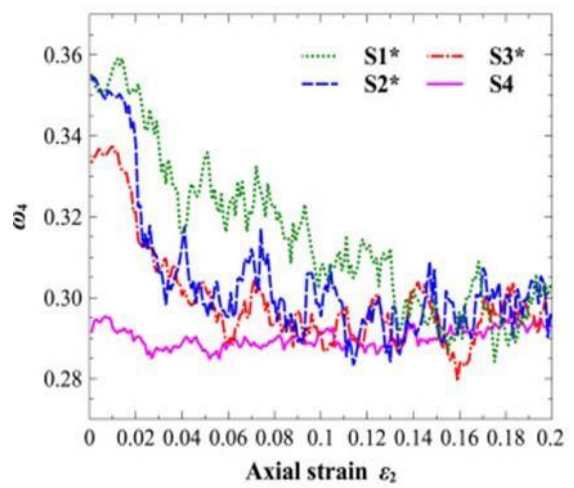

(c)

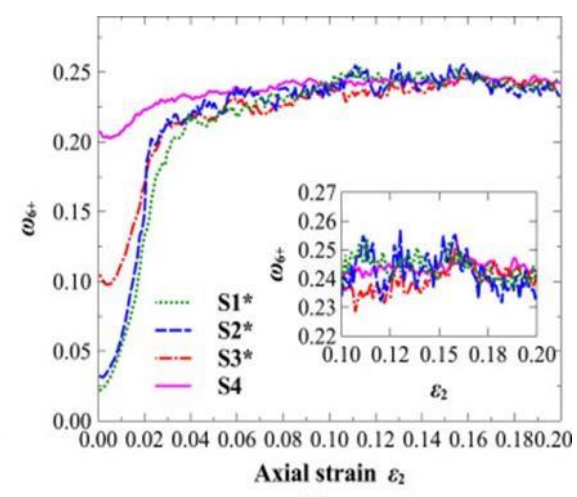

(b)

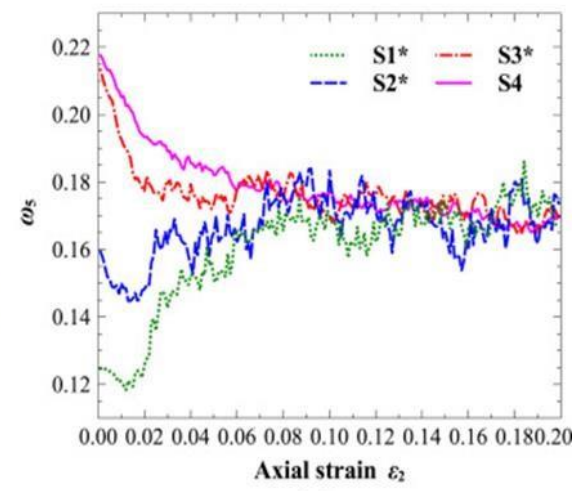

(d)

Fig. 4: comparison between the ratios of loops of 3 (Fig.(a)), more than 6 (Fig.(b)), 4 (Fig.(c)) and 5 grains (Fig.(d)) inside the shear bands (noted by a *) for dense samples S1, S2, S3 with the ratios of loops for the whole sample for the loose specimen S4 [9].

\section{A scenario for shear band formation: observations}

Same DEM code "YADE" as before has been used for the numerical results presented in this section, but with slightly different parameters (see [16]):

particle number: 20000; $\mathrm{D}_{50}=0.008 \mathrm{~m}$; uniform distribution of particles

$\mathrm{k}_{\mathrm{n}} / \mathrm{D}_{\mathrm{s}}=300 \mathrm{MPa} ; \mathrm{k}_{\mathrm{t}} / \mathrm{k}_{\mathrm{n}}=0.5$; intergranular friction angle $\varphi_{\mathrm{g}}=35^{\circ}$; initial porosity: 0.161

( $D_{s}=2 R_{1} R_{2} /\left(R_{1}+R_{2}\right)$ with $R_{1}$ and $R_{2}$ the radii of particles in contact)

confining pressure $\mathrm{p}_{\mathrm{a}}=100 \mathrm{kPa}$; loading rate: $0.01 \mathrm{~s}^{-1}$

In Fig. 5, two kinds of localized objects are observed on the maps of incremental deviatoric strains, as a biaxial loading is applied on a 2D numerical dense sample. The left figure corresponds to the initial state after an isotropic compression (State A in the stress-strain curves), where not any pattern of localization is visible for the first deviatoric computational increment. Then, very soon after the beginning of the biaxial compression (with a constant lateral stress) and until the maximum of contractancy, the second and third figures (Fig. 5, states B and C) show a network of meso-localised bands (involving tens of grains). At the stress peak (Fig. 5, state D), two macro-localized bands are clearly visible, giving rise to a unique band on the fifth figure at the end of loading. Besides, the fluctuating boundaries of shear bands in Fig. 5 are probably due to local fluctuations in stress states 
related to the rigid lateral boundary conditions. Indeed it is known that for flexible lateral boundaries the shear bands are more marked.
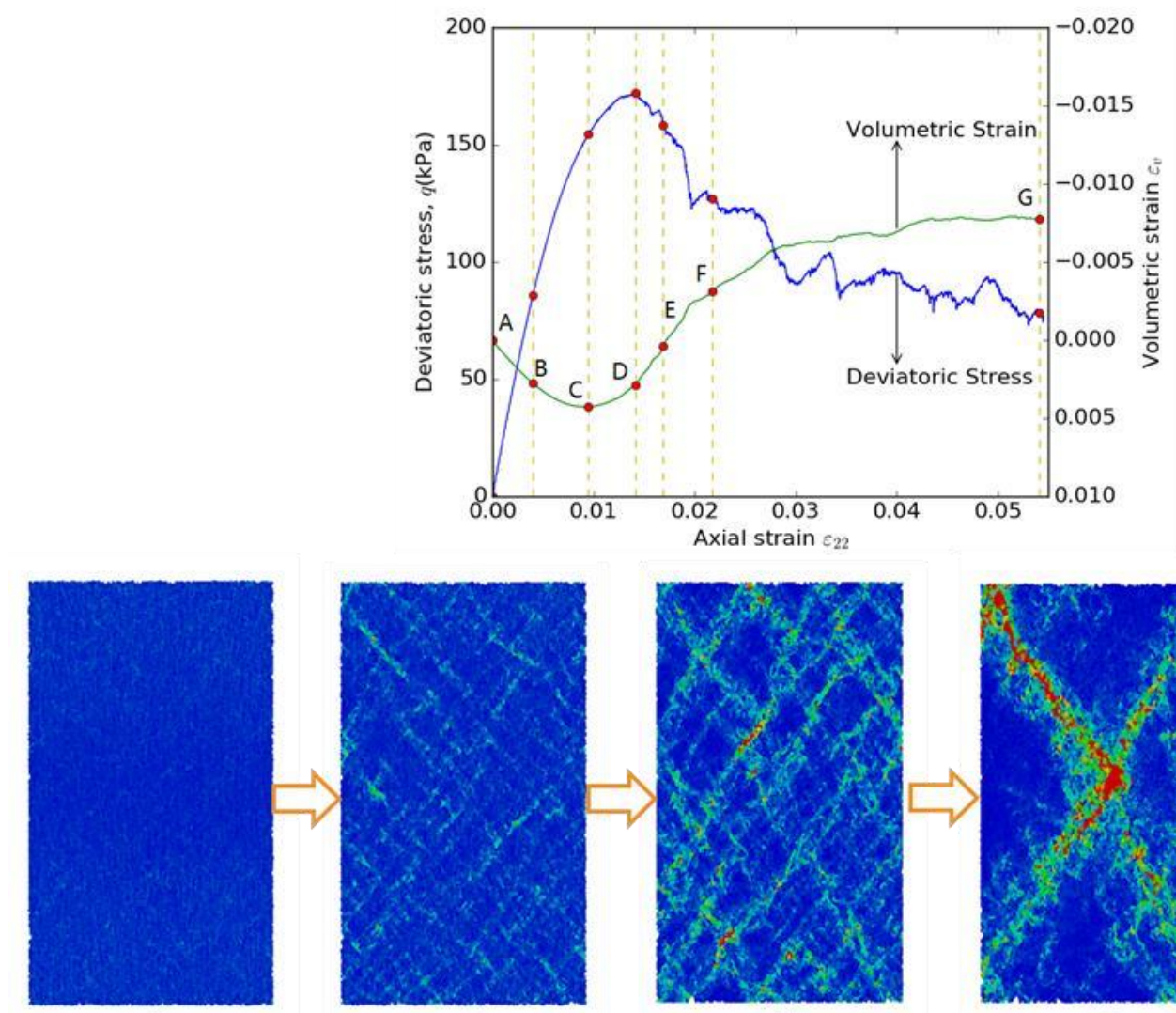

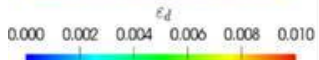

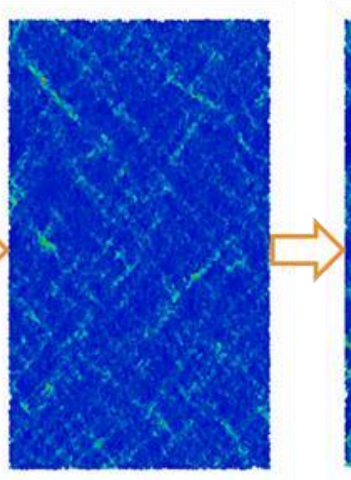

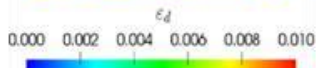

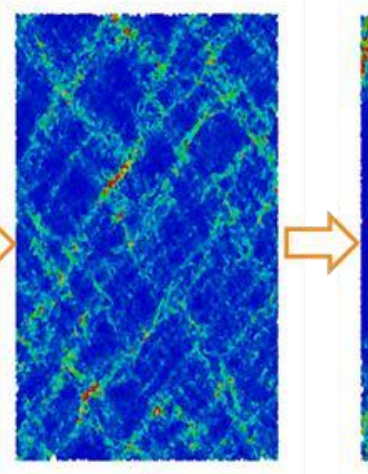

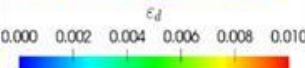
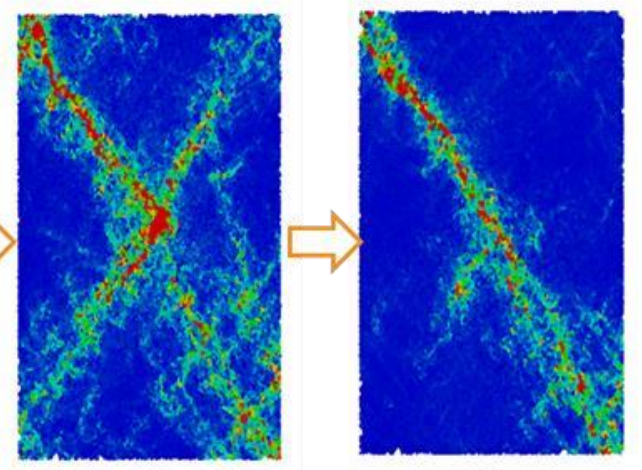

$0.000 \quad 0.002 \quad 0.004 \quad 0.000 \quad 0.008 \quad 0.010$

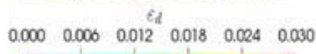

Fig. 5: Dense case: transition from a network of meso-slip lines into few macro-shear bands. The stress-strain and volumetric strain curves on the top indicate five characteristic states in the stressstrain evolution: A,B,C,D and E respectively [16]. Incremental deviatoric strain maps are shown for these five states.

In the loose case, the same obervations hold but with different mobilized friction and dilatancy angles. The change of sign in the dilatancy angle (see Fig.2) may help to understand qualitatively which of the friction or dilatancy angle drives the most the direction of the meso-slip lines. As shown in previous papers [6, 14] and in Fig. 6, for loose initial densities a diffuse mode of failure can persist without any macro shear band. However a network of meso localized bands is still observed. These bands are larger than in the dense case, but also intermittent and fluctuating during the loading. Their boundaries are not so well defined as in the dense case, probably because of the rigid boundaries. Eventually let us note that this localization network appears for very small deviatoric strains, i.e. for the first incremental strain (from the initial isotropic state) used to compute the incremental deviatoric strain map (see sub-figure on the left of fig. 6). 

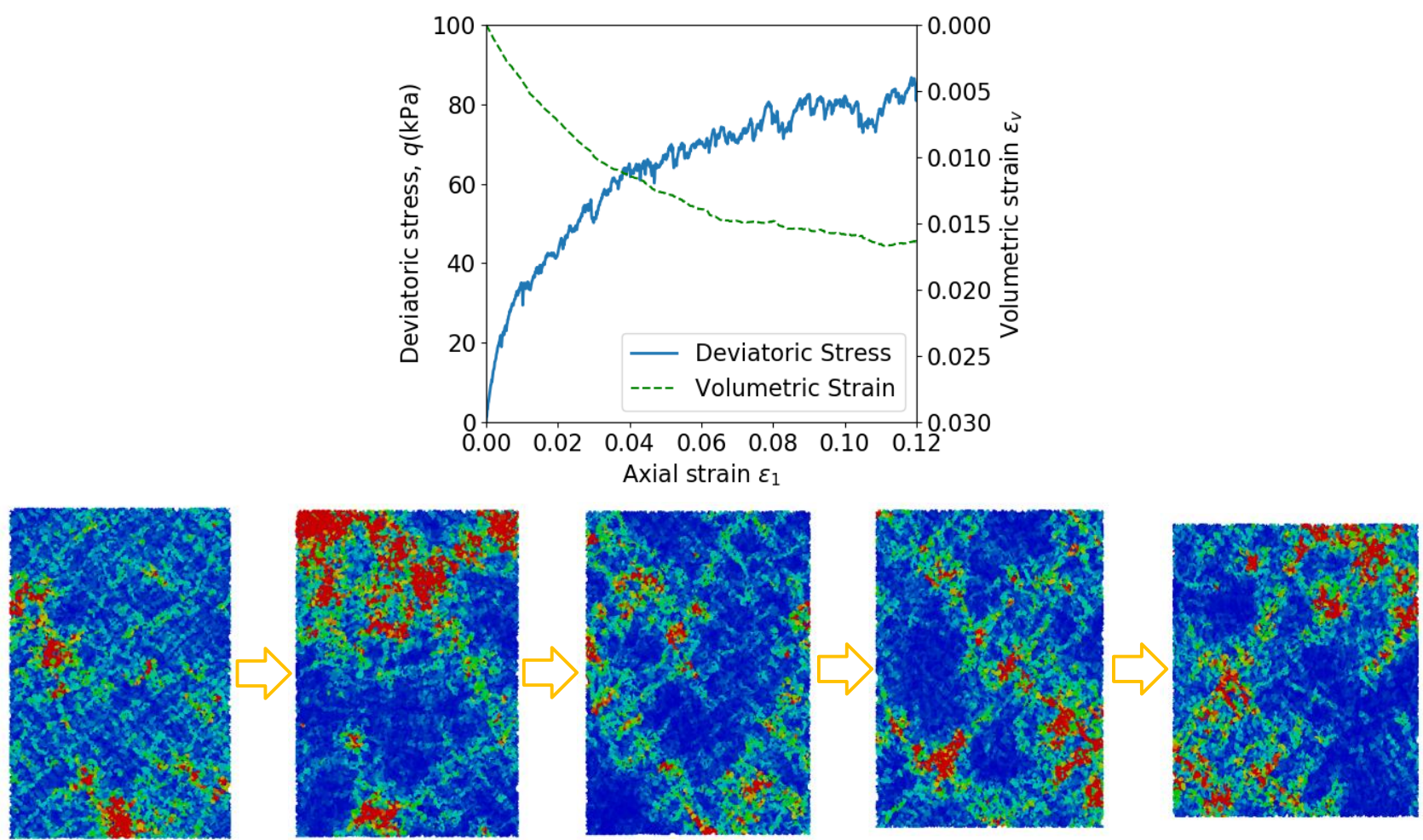

Fig. 6: Loose case. A diffuse mode of failure is persistent without any shear band. Only some networks of meso-slip lines are observed on these deviatoric strain maps plotted for axial strains equal to 0 ., $0.01,0.02,0.04,0.12$.

In summary, in the dense case (fig.5), the first appearing meso-network is constituted by thin, intermittent, fluctuating and small (with respect to the sample characteristic length) localized bands [22]. On the other hand, the second localization pattern consists of few thick and rather permanent macro localized bands developing at the sample scale. In the loose case (fig.6), only the first mesonetwork is observed, which is characteristic of a diffuse mode of failure without any macro bands. The meso-network is not as thin as in the dense case but is still intermittent, fluctuating, of small length and appearing as soon as the first incremental deviatoric strain is applied.

\section{A scenario for shear band formation: analysis}

Let us now analyse these two different localized objects by taking into account the available theories.

The second localization pattern, observed in the dense case, has been extensively studied [1, 2, 3, 11, $12,13,23$ ] and corresponds to shear bands, as described by localization criterion (5). Indeed this criterion gives, with a good predictive capacity, reasonable values of the critical stress states, where shear bands are detected experimentally slightly before the plastic limit condition ( $\operatorname{det} \boldsymbol{M}=0$, where $\mathbf{M}$ is the $6^{*} 6$ elasto-plastic matrix) for non-associate materials like the granular media. The shear band orientation is also well predicted and depends essentially on elasto-plastic tensor $\boldsymbol{L}$ (if boundary conditions influence is neglected). As $\boldsymbol{L}$ is not easy to compute in practice, no explicit theoretical value for this orientation can be obtained from this kind of shear band analysis, and empirical relation (2) is often used instead. 
Because the first meso-network of small localized bands appears from the very beginning of the biaxial loading (state A in Fig. 5 and first sub-figure on the left in Fig. 6), it cannot be interpreted as shear bands. Indeed, the localization criterion (5) cannot be verified so far from the plastic limit condition (peak state D in Fig. 5 and stress plateau in Fig.6), even if the localization criterion can be fulfilled slightly before the plastic limit condition (given by det $\mathbf{M}=0$ ) for non-associate materials like granular media ([11]).

As a result, since large incremental strain is known to be a signature of plasticity, we propose to interpret the lines of high incremental deviatoric strains as slip lines as described in the plasticity theory. Fully independent on shear band theory, these so-called slip lines are thus obtained from perfect plasticity theory $[17,18,19]$ (also called limit analysis theory).

The rate-independent solid body is assumed to be rigid-plastic, elastic strains being supposed negligible with respect to plastic strains. In soil mechanics the plasticity yield criterion, which has been well verified experimentally in 2D and axisymmetric conditions for purely frictional granular media, is the Mohr-Coulomb's criterion given by:

$$
\left(\sigma_{1}-\sigma_{3}\right) /\left(\sigma_{1}+\sigma_{3}\right)=\sin \varphi
$$

with $\varphi$ being the internal friction angle of the material ( see fig.1).

So, if it is assumed that the whole body is at a plastic state, the stress state satisfies equation (7). By considering equation (7) together with the equilibrium equations and by taking into account boundary and initial conditions, an hyperbolic set of equations is obtained whose solutions give characteristic lines, called « slip lines ». These solutions are of course of a fully different nature from elastic solutions, which correspond to an elliptic set of equations.

In the simple case of a biaxial compression, the orientation of the slip lines with respect to the major stress direction (the vertical direction in the figures) is given by $\pi / 4-\varphi / 2$ (including the symmetric lines with respect to this stress direction). For biaxial extensions, the orientation with respect to the same vertical direction is $\pi / 4+\varphi / 2$ (and the symmetric lines). These directions correspond to surfaces where the ratio between the shear stress over the normal stress is maximal.

These results are obtained by assuming that the plastic strains are associated (friction angle $\varphi$ is equal to dilatancy angle $\psi$ ). However for granular materials these two angles are different by $10^{\circ}$ to $15^{\circ}$ according to classical experimental results $\left(\psi=\varphi-10^{\circ}\right.$ to $\left.15^{\circ}\right)$. As a result, for non-associated materials the direction of the slip lines is predicted by either the friction angle (static solution derived by Mohr-Coulomb yield surface) or the dilatancy angle (kinematic solution obeying to the material flow rule).

The kinematic solution corresponds to a plastic strain satisfying (see fig.1):

$$
\left(\mathrm{d} \varepsilon_{1}+\mathrm{d} \varepsilon_{3}\right) /\left(\mathrm{d} \varepsilon_{1}-\mathrm{d} \varepsilon_{3}\right)=\sin \psi
$$

Then, the kinematic direction of the slip lines is given by material elements subjected to a ratio between angular distorsion $\left(d \varepsilon_{1}-d \varepsilon_{3}\right) / 2$ and relative volumetric variation $\left(d \varepsilon_{1}+d \varepsilon_{3}\right) / 2$ satisfying equation (8). The material direction with respect to the major stress direction (the vertical direction in the figures) in case of biaxial compression is then $\pi / 4-\psi / 2$ (including the symmetric lines with 
respect to this stress direction). For biaxial extensions, the orientation with respect to the same vertical direction is $\pi / 4+\psi / 2$ (and the symmetric lines).

To summarize, when $\psi=\varphi$, the normality of the flow rule is fulfilled and the incremental plastic strain is normal to the Mohr-Coulomb yield surface. However, for granular media, $\psi \neq \varphi$, and the limit analysis gives two possible solutions for the macro-slip line orientation in an ideal perfectly homogeneous scale:

$$
\begin{aligned}
& \pi / 4-\varphi / 2 \text { in static analysis, } \\
& \pi / 4-\psi / 2 \text { in kinematic analysis. }
\end{aligned}
$$

Let us now apply this perfect plasticity theory, developed in an homogeneous continuum mechanics framework, to the present discrete granular medium, essentially heterogeneous at meso-scale. At this scale, the macro-friction and macro-dilatancy angles are no more the proper mechanical parameters. So the directions of slip lines are governed either by the meso-yield criterion (described by the mobilized internal friction angle at the meso-scale, when using the Mohr-Coulomb criterion) or by the meso-flow rule (described by the mobilized dilatancy angle at the meso-scale). These two directions do not coincide in case of non-associated plasticity and they have to be estimated from the mesoscale behavior and not directly from macroscale homogenized friction and dilatancy angles. Presently, a specific and consistent definition of friction and dilatancy angles at mesoscale remains still a challenge which is out of the scope of the present paper.

It appears that the macro-shear band orientation estimated by equation (2) and the meso-slip line orientation given by equations (9) and (10) are very close each other, even though they stem from fully independent theories: elasto-plasticity for shear bands and rigid-perfect plasticity for slip lines. This comment is in line with the observations in Fig. 5 where the orientation of the first appearing network of meso-slip lines is close to the orientation of the few macro-shear bands appearing around the failure state. Let us note that, while the definition of slip line network is perfectly clear from a continuum mechanics point of view as recalled here, its discrete signature in a discrete mechanics framework still need some work and is postponed to forthcoming papers, where in particular the orientations of slip lines and shear bands will be compared quantitatively.

Let us have in mind eventually that sliding directions are given by the local orientation of contacts and do not incorporate any geometrical effect (such as grain loop deformation). This results in the fact that sliding directions are different from slip line directions. The present preliminary quantitative analysis of sliding directions shows that contacts slide mostly in the vertical direction in drained biaxial test (contact normals perpendicular to the principal stress direction), while slip lines are oriented in a direction close to $50^{\circ}$ with respect to the horizontal direction.

A complete picture of the failure process in granular media is thus emerging with the following trends:

- From the very beginning of the loading, a network of meso-slip lines appears due to local granular plastic or geometric instabilities, always present in any microstructurally heterogeneous material as a function of the imperfect initial state and boundary conditions. For a macro-perfectly homogeneous sample subjected to uniform boundary conditions, these slip lines are straight lines whose orientation with respect to the major principal stress 
direction is given either by equation (7) or by equation (8). But the typical length scale characterizing the network of meso-slip lines remains much smaller than the sample dimension, which makes them largely independent on boundary conditions. The meso-slip line orientation is thus essentially function of both the material plastic mechanical mesoproperties and its initial density.

- For some initial densities and some loading paths (this point is discussed later on), this mesonetwork is fluctuating but persistent at large strains. Not any macro-localization pattern can be observed. The failure mode can be characterized as diffuse - see for example Fig. 1(d) and Fig.6. Typically, this is the case for loose sands, or even dense sands if the strain loading path imposes a large enough dilatancy.

- For other initial densities or other loading paths, some critical slip lines (a discussion follows about the definition of "critical”) give rise to shear bands just before the plastic limit condition (for non-associated materials where $\operatorname{det} \boldsymbol{n}^{t} . \boldsymbol{L} . \boldsymbol{n}$ vanishes before $\operatorname{det} \boldsymbol{M}$ ) or at the plastic limit for associated materials. These macro-bifurcations of the strain mode are described by the localization criterion recalled in equation (5), and the orientation of the shear bands (or compaction/dilation bands) is given by the perpendicular direction to vector $\boldsymbol{n}$ solution of equation (5), when real solutions do exist. Let us note that shear bands eventually depend partly on boundary conditions because the typical length scale characterizing shear bands is of the same order of magnitude as the usual sample dimension. The sample aspect ratio favors or delays the shear band formation. Plane strain conditions favor the existence of shear bands, whereas they can vanish in plane stress conditions. Moreover flexible (through the presence of membranes) or rigid boundary conditions modify the shear band propagation with possible reflections on a rigid wall. Often, corners or other geometric singularities play the role of initiators or attractors for respectively the rising or the propagation of shear bands. Overall, boundary conditions modify slightly the orientation of shear bands as seen in Fig.1 and 5 (see more examples in [14]). Let us insist once more that this is not at all the case for the slip line network which is developing at meso scale.

An ultimate question remains, concerning the condition under which a network of meso-slip lines becomes "critical” and gives rise to a macro-shear band. This question is still widely open and it will be investigated in details in forthcoming papers. In the present paper, it is conjectured that a slip line is bifurcating into a shear band when meso-softening occurs. This results in local modifications of the tangent modulus eigenvalues from positive to negative values. Then, a bifurcation of the macro-strain mode arises, transforming the critical meso-slip line into a macro-shear band as it is for example observed in Fig.5. On the other hand, if the local material behavior does not present any softening (following the local density and the present local incremental loading direction), the occurrence of a slip line does not present any "catastrophic" nature (in the sense of the "catastrophe theory" by Thom [20]). The slip line gives rise to locally stable meso-localized strains and not any macro-bifurcation is expected from this local material or geometric meso-bifurcation.

\section{Conclusion}


According to the observations from Fig. 1 and 3 to 6, we can distinguish two interacting localized strain structures that are different in nature: meso-slip lines and macro-shear bands. Meso-slip lines are narrow, thin and small structures randomly distributed within the whole granular sample, and their orientations are given by limit analysis theory. Since the granular assembly is equivalent to a contact network, the meso-slip lines can be seen as connected shear structures joining sliding contacts. These meso structures are supposed to undergo large deviatoric strains for the few of them which are critical as observed in Fig. 5 and 6. While this concept of shear strain chain will be introduced with more details in further papers, it can be noted here that the meso-slip lines own unique features during loading. They fulfill the local criterion of plasticity (according to the static limit analysis) or the local flow rule (according to the kinematic limit analysis) or probably a more complex criterion for nonassociated anisotropic materials as granular media. Besides, some meso-slip lines could bifurcate into macro-shear bands once Rice's localization criterion (eqn (5)) is fulfilled, while outside the shear band the meso-slip line network disappears, as observed in Fig. 1 and 5, due to elastic unloading. In other words, few critical slip lines concentrate into shear bands. Let us note also that the shear band boundaries are fluctuating, as observed in Fig. 1 and 5, with many meso exchanges (probably accentuated here by the rigid lateral boundary conditions), while the meso-slip lines evolve by local contact sliding and contact losses and gains.

Eventually the following scenario is conjectured. According to catastrophe [20] and perfect plasticity $[17,19]$ theories, there is a first global bifurcation of the strain mode giving rise to a network of mesoslip lines. The orientation of these meso-slip lines corresponds to non-associated perfect plasticity solutions (eqns (9) and/or (10)). Then a second bifurcation may appear for few critical slip lines, due to a meso-softening leading to the fulfilling of the macro-localization criterion (eqn (5)). So, mesoslip lines concentrate around the location of this meso-softening giving rise to macro-shear bands with an orientation corresponding to the localization criterion (eqn (5)) together with the influence of the boundary conditions. In this scenario, the shear band contains a dense network of meso-slip lines, what is fully consistent with the existence of a unique identical microstructure at failure inside shear band for dense granular materials and in the whole material domain for loose granular media, as shown in section 2. Basically, this paper proposes to analyze both the different localized objects observed in DEM simulations in the general framework of non-associated elasto-plasticity. It has leaded to build an interpretation of the failure mechanisms in granular media in terms of meso-slip lines and macro-shear bands. In the end, a conjecture is formulated to explain the transition sometimes but not always - from slip lines to shear bands. This scenario will be discussed and questioned quantitatively in forthcoming papers.

\section{References}

[1] I. Vardoulakis, M. Goldscheider, G. Gudehus, Formation of shear bands in sand bodies as a bifurcation problem, Int. J. Num. Anal. Meth. Geomech., 2, 99-128 (1978)

[2] I. Vardoulakis, Shear band inclination and shear modulus of sand in biaxial tests, Int. J. Num. Anal. Meth. Geomech., 4, 103-109 (1980)

[3] J. Desrues, Shear band initiation in granular materials: experimentation and theory, in 
Geomaterials Constitutive Equations and Modelling, F. Darve ed., Elsevier (1990) and Taylor and Francis publ. (2002)

[4] M.R. Kuhn, Structured deformations in granular8] materials, Mech. of Mat., 31(6), 407-429 (1999)

[5] F. Darve, X. Roguiez, Homogeneous bifurcation in soils, in Localization and Bifurcation Theory for Soils and Rocks, Adachi Oka and Yashima eds, Balkema publ. (1998)

[6] F. Darve, G. Servant, F. Laouafa, Failure in geomaterials: continuous and discrete analyses, Comp. Meth. in Appl. Mech. and Eng., 193 (27-29), 3057-3085 (2004)

[7] F. Darve, Liquefaction phenomenon: modelling, stability and uniqueness, in Verification of Numerical Procedures for the Analysis of Soil Liquefaction Problems, Arulanandan and Scott eds, Balkema publ. (1993)

[8] L. Sibille, P. Villard, F. Darve, R. Aboul Hosn, Quantitative prediction of discrete element models on complex loading paths, Int. J. Num. and Anal. Meth. in Geomech., 43(5), 858-887 (2019)

[9] H. Zhu, H.N.G. Nguyen, F. Nicot, F. Darve, On a common critical state in localized and diffuse failure modes, J. Mech. Phys. Solids, 95, 112-131 (2016)

[10] J. Desrues, C. Viggiani, Strain localization in sand, an overview of the experimental results obtained in Grenoble using stereophotogrammetry, Int. J. Num. and Anal. Meth. in Geomech., 28(4), 279-321 (2004)

[11] J. Rice, The localization of plastic deformation, in Theoretical and Applied Mechanics, W.T. Koiter ed., North Holland publ., 207-220 (1976)

[12] J. Mandel, Condition de stabilité et postulat de Drucker, in Rhéologie et Mécanique des Sols, Kravtchenko and Sirieys eds, Springer publ. (1966)

[13] F. Darve, An incrementally non-linear constitutive law of the second order and its application to localization, in Mechanics of Engineering Materials, C.S. Desai and R.H. Gallagher eds, Wiley, 179196 (1987)

[14] R. Wan, F. Nicot, F. Darve, Failure in Geomaterials a Contemporary Treatise, Iste-Elsevier (2017)

[15] K. Hamadi, A. Modaressi, F. Darve, Bifurcation and instability modelling by a multimechanism elasto-plastic model, Int. J. Num. Anal. Meth. In Geomech., 32(5),461-492 (2008)

[16] J. Liu, F. Nicot, Z. Wei, Sustainability of internal structures during shear band forming in 2D granular materials, Powder and Techn. , 338, 458-470 (2018)

[17] R.O. Davis, A.P.S. Selvadurai, Plasticity and Geomechanics, Cambridge Univ. Press (2012)

[18] J.R. Rice, Plane strain slip line theory for anisotropic rigid/plastic materials, J. Mech. Phys. Solids, 21, 6318-74 (1972) 21

[19] H.-S. Yu, Plasticity and Geotechnics, Springer (2006) 
[20] R. Thom, Stabilité Structurelle et Morphogénèse: Essai d'une Théorie Générale des Modèles, Ediscience S.A. (1972)

[21] J. Liu, A. Wautier, S. Bonelli, F. Nicot, F. Darve, Macroscopic softening in granular materials from a mesoscale perspective, Int. J. Solids Structures, accepted (2020)

[22] Le Bouil, A., Amon, A., McNamara, S., \& Crassous, J. (2014), Emergence of cooperativity in plasticity of soft glassy materials, Physical review letters, 112(24), 246001.

[23] Desrues, J., Andò, E., Mevoli, F. A., Debove, L., \& Viggiani, G. (2018), How does strain localise in standard triaxial tests on sand: Revisiting the mechanism 20 years on, Mechanics Research Communications, 92, 142-146. 